\title{
Duodenal Stump Reinforcement with Purse-string Suture in Distal Gastrectomy under Laparoscope Combined with Uncut Roux-en-Y Reconstruction in Patients with Gastric Cancer
}

\section{Fengyuan Li}

The First Affiliated Hospital of Nanjing Medical University

Jianghao Xu

The First Affiliated Hospital of Chongqing Medical University

Hao Xu

The First Affiliated Hospital of Nanjing Medical University

\section{Weizhi Wang}

The First Affiliated Hospital of Nanjing Medical University

\section{Diancai Zhang}

The First Affiliated Hospital of Nanjing Medical University

\section{Li Yang}

The First Affiliated Hospital of Nanjing Medical University

\section{Linjun Wang}

The First Affiliated Hospital of Nanjing Medical University

\section{Yugang Ge}

The First Affiliated Hospital of Nanjing Medical University

\section{Yiwen Xia}

The First Affiliated Hospital of Nanjing Medical University

\section{Liang Shi}

The First Affiliated Hospital of Nanjing Medical University

\section{Lu Wang}

The First Affiliated Hospital of Nanjing Medical University

\section{Zekuan Xu ( $\sim 1963278799 @ q q . c o m)$}

First Affiliated Hospital of Nanjing Medical University https://orcid.org/0000-0002-0426-2169

\section{Research}

Keywords: Duodenal Stump Reinforcement, laparoscopy, Uncut Roux-en-Y Reconstruction, gastric cancer

Posted Date: October 26th, 2020 
DOI: https://doi.org/10.21203/rs.3.rs-94552/v1

License: (a) (i) This work is licensed under a Creative Commons Attribution 4.0 International License. Read Full License 


\section{Abstract}

Background: This study aimed to compare patient outcomes after laparoscopic distal gastrectomy with uncut Roux-en-Y reconstruction for gastric cancer between a group who underwent a duodenal stump reinforcement procedure and those who did not.

Methods: Data from 233 patients with gastric cancer (GC) undergoing distal gastrectomy under laparoscope combined with uncut Roux-en-Y reconstruction were retrospectively investigated. Patients were divided into two groups. The non-reinforcement group (NR) underwent surgery from June 2014 to March 2015 with no reinforcement of the duodenal stump $(n=54)$ and the reinforcement group $(R)$ underwent surgery from April 2015 to June 2018 with reinforcement of the duodenal stump ( $n=179)$. In group $\mathrm{R}$, the duodenum was divided using an endoscope-assisted linear stapler, which was reinforced by a purse-string suture along the duodenal staple line.

Results: Duodenal stump leakage was observed in 2 patients from group NR (3.7\%), while no duodenal stump leakage or fistula was detected in group R. In addition, no significant difference was observed in the patient characteristics between group NR and $\mathrm{R}$.

Conclusions: The incidence of duodenal stump leakage can be reduced by reinforcement with a pursestring suture.

\section{Background}

Duodenal stump fistula (DSF) is a rare but serious adverse event observed in gastric cancer (GC) patients following gastrectomy with Roux-en-Y or Billroth II reconstruction ${ }^{[1,2]}$. Clinically, the common complications of duodenal stump leakage include peritonitis, abdominal sepsis, bleeding, multiorgan failure, and death. In recent years, Roux-en-Y reconstruction has been extensively carried out for the sake of reducing bile reflux after distal or total gastrectomy in GC patients; moreover, the linear stapler has been employed to cut the duodenum in many cases. It has been reported that most duodenal stump staple-line leaks are related to ischemic issues or mechanical issues, including intraluminal pressures that exceed the tolerance of the tissue and suture-line ${ }^{[3-5]}$. Typically, the staple line is reinforced by sutures in conventional open surgery.

The first case of distal gastrectomy under laparoscope was carried out by Kitano et al in 1994 ${ }^{[6]}$. In recent decades, open gastric surgery has been gradually substituted by a laparoscope-assisted operation, which can be ascribed to the remarkable short-term benefits of the latter, including lower pain, smaller wound, decreased blood loss, as well as accelerated recovery. The duodenal stump can be easily reinforced in open surgery compared with laparoscopic surgery, but it is not always carried out in the latter. Recently, great achievements have been made in laparoscopic suturing techniques and training methods ${ }^{[7]}$, which makes it possible for surgeons who are proficient at intracorporeal knot tying to reinforce the duodenal staple line and reduce the risk for duodenal stump leakage ${ }^{[8]}$. Herein, a duodenal stump reinforcement 
procedure is reported in distal gastrectomy under laparoscope plus Roux-en-Y reconstruction. The surgical procedures are shown in a video and the short-term surgical outcomes are reported as follows.

\section{Methods}

\section{Ethics Statement}

The study was approved by the institutional review board of Nanjing Medical University. Informed written consent was obtained from all subjects. The experimental protocol was carried out in accordance with the approved guidelines.

\section{Study Population}

Between September 2014 and June 2018, data from GC patients receiving uncut Roux-en-Y reconstruction after laparoscopic distal gastrectomy at the Department of Gastric Surgery of the First Affiliated Hospital of Nanjing Medical University were retrospectively analyzed. In our institution, uncut Roux-en-Y reconstruction had become the most frequently employed surgical procedure following laparoscopic distal gastrectomy. The enrolled patients were divided into 2 groups. The non-reinforcement group (NR) included patients who underwent gastrectomy with no reinforcement of the duodenal stump but with a purse-string suture from September 2014 to August $2015(n=54)$. The reinforcement group (R) included patients who underwent gastrectomy with reinforcement of the duodenal stump from September 2015 and June $2018(n=179)$. All patients received laparoscopic distal gastrectomy by the same group of surgeons.

\section{Surgical Procedure}

First, the camera port was prepared in or beneath the umbilicus with the patients in supine position and both legs apart. For all patients, 5 trocars were used for the surgical procedure, as described in Fig. 1. Lymph node dissection (LND) was carried out in accordance with the guidelines from the Japanese Gastric Cancer Association. ${ }^{[9]}$ Afterwards, the duodenum was divided with an endoscopic linear stapler (Echelon 60, white cartridge; Ethicon Endo Surgery Co.). For this, the surgeon stood at the patient's left side, while the camera assistant stood between the patient's legs., The duodenum was cut using a $60 \mathrm{~mm}$ endoscope-assisted linear stapler through the trocar in the left upper side with a single white cartridge. In addition, in group $\mathrm{R}$, the duodenal staple line would be reinforced by a hand-sewn purse-string suture (continuous muscular absorbable 3-0 sutures) (Fig. 1 and Supplemental Digital Content 1).

Clinicopathological characteristics, surgical outcomes, and postoperative complications of all patients were extracted from the hospital database and the electronic medical record of each patient. Hospital mortality was defined as death within 30-days or anytime during the postoperative hospitalization. The gastric cancer stage was classified according to American Joint Committee on Cancer (AJCC) stage. 


\section{Statistical Analysis}

The JMP program (SAS Inc., Cary, NC) and R version 9.0.0 was used for all statistical analyses. All continuous variables are presented as mean \pm standard deviation (SD). Differences with a two-sided $P$ value $<0.05$ were considered statistically significant. The $\chi 2$ test was applied to assess the differences in proportions. Differences in age, body mass index (BMI), harvested lymph node number, operation time, blood loss, and length of hospital stay between the two groups were assessed using the Student $t$ test.

\section{Results}

Of the total participants, 179 received reinforcement of the duodenal stump between September 2015 and June 2018 (R group) and 54 underwent duodenal cut alone without reinforcement between September 2014 and August 2015 (NR group). Table 1 summarizes the clinical features of all patients. According to analysis, no statistically significant difference was observed between group NR and R in age, gender, BMI, tumor size or American Joint Committee on Cancer (AJCC) stage. Table 2 shows the operation outcomes as well as clinical course after surgery for both groups. Differences in operation time, blood loss, harvested lymph node number, first flatus, and liquid diet between the two groups were not statistically significant. The time before first flatus was longer in group NR than in group R [75.03 \pm 25.69 vs. $67.45 \pm 21.88, P=0.033$ ] and the postoperative length of stay was also longer in group NR than in group R [9.85 \pm 3.48 vs. $8.42 \pm 1.99, \mathrm{P}<0.001]$. Duodenal stump leakage was seen in 2 cases from group NR (Table 3). No deaths due to abdominal sepsis, bleeding, or multiorgan failure associated with duodenal stump leakage was reported. No patient had duodenal stump leakage or fistula in group R. In the NR group, 1 patient had a chylous fistula and 3 patients developed bleeding due to gastrointestinal anastomosis, 1 had gastric atony, 1 had intra-abdominal hemorrhage, and 1 had other complications (such as fever or pulmonary inflammation). In group $\mathrm{R}, 1$ patient had bleeding due to gastrointestinal anastomosis, while 3 patients had other complications (such as fever or pulmonary inflammation). 
Table 1

Clinicopathological characteristics of patients (n (\%)).

\begin{tabular}{|c|c|c|c|}
\hline Items & $N R(n=54)$ & $R(n=179)$ & $\mathbf{P}$ \\
\hline Age(years) & $58.19 \pm 10.89$ & $58.91 \pm 10.10$ & 0.652 \\
\hline \multicolumn{4}{|l|}{ Gender } \\
\hline Male & $34(63.0)$ & $117(65.4)$ & 0.746 \\
\hline Female & $20(37.0)$ & $62(34.6)$ & \\
\hline $\mathrm{BMI}(\mathrm{kg} / \mathrm{m} 2)$ & $23.00 \pm 3.86$ & $23.22 \pm 2.87$ & 0.567 \\
\hline Tumor size(cm) & $2.61 \pm 1.54$ & $2.53 \pm 1.72$ & 0.081 \\
\hline AJCC pTNM stage & $39(72.2)$ & 107(59.8) & 0.243 \\
\hline$I A+I B$ & $9(16.7)$ & $40(22.3)$ & \\
\hline$\|A+\| B$ & $6(11.1)$ & $32(17.9)$ & \\
\hline \multicolumn{4}{|l|}{$I I I A+I I I B+I I I C$} \\
\hline \multicolumn{4}{|c|}{$\begin{array}{l}\text { Values are presented as mean } \pm \text { SD, number only, or number (\%). NR, Not Reinforced(September } 2014 \\
\text { to August 2015); }\end{array}$} \\
\hline \multicolumn{4}{|c|}{$\begin{array}{l}\text { R, Reinforce(September } 2015 \text { to December 2016); BMI, body mass index; AJCC, American Joint } \\
\text { Committee on Cancer 7th; pTNM, pathological tumor node metastasis. }\end{array}$} \\
\hline
\end{tabular}

Table 2

Operative findings and postoperative clinical course (n).

\begin{tabular}{|llll|}
\hline Items & $\mathbf{N R}(\mathbf{n}=\mathbf{5 4})$ & $\mathbf{R}(\mathbf{n}=179)$ & $\mathbf{P}$ \\
\hline Operation time $(\mathrm{min})$ & $166.27 \pm 25.13$ & $183.08 \pm 34.25$ & $<0.001$ \\
\hline Blood loss $(\mathrm{mL})$ & $51.07 \pm 42.74$ & $49.01 \pm 25.00$ & 0.739 \\
\hline Retrieved lymph nodes & $37.00 \pm 10.93$ & $37.70 \pm 11.55$ & 0.694 \\
\hline First flatus (hours) & $75.03 \pm 25.69$ & $67.45 \pm 21.88$ & 0.033 \\
\hline Liquid diet (days) & $5.07 \pm 0.93$ & $5.02 \pm 1.11$ & 0.756 \\
\hline Postoperative hospital stay (days) & $9.85 \pm 3.48$ & $8.42 \pm 1.99$ & $<0.001$ \\
\hline $\begin{array}{l}\text { Values are presented as mean } \pm \text { SD. NR, Not Reinforced(September 2014 to August 2015);R, Reinforce } \\
\text { (September 2015 to December 2016) }\end{array}$ & & \\
\hline
\end{tabular}


Table 3

Postoperative complications ( $\mathrm{n}(\%))$.

\begin{tabular}{|llll|}
\hline Items & $\mathbf{N R}(\mathbf{n}=\mathbf{5 4})$ & $\mathbf{R}(\mathbf{n = 1 7 9 )}$ & $\mathbf{P}$ \\
\hline Duodenal stump leakage & 2 & 0 & 0.053 \\
Chylous fistula & 1 & 0 & 0.232 \\
\hline Intra-abdominal hemorrhage & 1 & 0 & 0.232 \\
Anastomotic bleeding & 3 & 1 & 0.040 \\
\hline Gastric atony & 1 & 0 & 0.232 \\
others & 1 & 3 & 1.00 \\
\hline Overall postoperative complications $(\mathrm{n}(\%))$ & $9(16.7)$ & $4(2.2)$ & $<0.001$ \\
\hline Postoperative mortality & 0 & 0 & \\
(n(\%)) & & & \\
\hline
\end{tabular}

\section{Statistical Analysis}

All continuous variables are presented as mean $\pm S D$. A 2sided P-value of $<0.05$ was considered statistically significant. The $\mathrm{X}^{2}$ test was applied to assess differences in proportions, and the Student $\mathrm{t}$ test was used to assess the difference in age, body mass index (BMI), dissected lymph nodes, operative time, bleeding, and hospital stay between groups.

\section{Discussion}

This study aimed to compare patient outcomes after laparoscopic distal gastrectomy with uncut Rouxen-Y reconstruction between a group who underwent a duodenal stump reinforcement procedure and those who did not. Our results show that duodenal stump leakage can be avoided by reinforcement with a hand-sewn purse-string suture.

At the institution of the present study, uncut Roux-en-Y reconstruction after laparoscopic distal gastrectomy was initiated in 2014 and has been frequently performed since 2015 . Compared with other reconstruction methods after distal or total gastrectomy, such as Billroth $\otimes$ or Billroth $\otimes$, uncut Roux-en- $Y$ a reconstruction can positively prevent bile reflux into the residual stomach and esophagus. However, it is also associated with DSF, which is a critical complication following gastrectomy which varies widely in onset time, output, severity, recurrence risk, and outcomes ${ }^{[10-11]}$. A number of nonsurgical approaches are preferred in the case of DSF, especially for the percutaneous surgeries (e.g. drainage of abdominal abscess $^{[12]}$ and glue injection to obliterate fistula ${ }^{[13,14]}$ ) or the endoscopic surgeries (e.g. application of clips ${ }^{[15]}$, endoloops, glue ${ }^{[16]}$, or to close the DSF). However, at least one reoperation would be necessary 
in more than 1 out of 3 patients. Numerous published case reports as well as various surgical methods have revealed simple surgical therapeutic strategies. Usually, a reoperation is carried out at an early stage, which is often under an emergency condition due to acute sepsis. However, such surgical procedures have not been standardized since they depend on the concurrent complications, including digestive fistula and intraabdominal bleeding. Nonetheless, peritoneal cleaning together with abdominal drainage has laid the foundation of surgical treatment for DSF [17].

Nevertheless, prevention is always better than cure. Purse-string suture reinforcement of the duodenal stump in Roux-en-Y anastomosis began in our institution September 2015. Duodenal stump leakage was observed in 2 patients $(3.7 \%, n=54)$ in our study, while the rate of duodenal leakage reported in other studies ( $\mathrm{n}>30$ cases) was $0.6-3.2 \%{ }^{[18-25]}$. By contrast, no patient in group $\mathrm{R}$ had duodenal stump leakage or fistula $(0.0 \%, n=179)$, with no significant difference between the two groups $(P=0.053)$. Nonetheless, other postoperative complications were discovered, including chylous fistula, intraabdominal hemorrhage, anastomotic bleeding, and gastric atony. Our results suggested that the incidence rate of all the above-mentioned postoperative complications in the NR group were higher than that in $\mathrm{R}$ group $(16.7 \%$ vs $2.2 \%, \mathrm{p}<0.001)$, indicating that purse-string suture reinforcement of the duodenal stump may also improve other postoperative complications. In addition, our findings demonstrated that the time of first flatus and postoperative length of hospital stay in group $\mathrm{R}$ were shorter compared with the NR group based on longer operation time (166.76 \pm 22.19 vs $182.89 \pm$ $34.15 \mathrm{~min}, \mathrm{p}<0.001)$, and such heterogeneity might be ascribed to the higher complication rates in the NR group.

Most published papers have not described whether they have reinforced the duodenal stump. Jiang et al. [26] reported that the duodenum was divided using an endoscopic linear stapler, and duodenal stump rupture could be prevented by interrupted seromuscular sutures. In their study, 2 patients (8.7\%) experienced duodenal stump leakage and the authors indicated that the duodenal stump leakage was caused by the torsion of $Y$ anastomosis. They also pointed out that the risk of such torsion would be reduced by the strict closure of the jejunum mesenterium and Petersen cavity. Moreover, the authors concluded that laparoscopic Roux-en-Y reconstruction should be carried out carefully in the hands of experienced laparoscopic surgeons. In our study, the Petersen and mesojejunal defects were closed.

There are several methods for duodenal stump reinforcement. In our institution, the purse-string suture was employed and was an important factor in the duodenum cutting process. For the present study, the duodenum was typically cut from the left side to the right side, which made it easy to invert the duodenum staple line. By contrast, without plans for reinforcement the duodenum would usually be cut through the trocar of the left-upper abdomen. Another important technique of our procedure involved the application of the same trocar in the left-upper abdomen for endoscopic linear stapler and needle forceps. This technique produced an easy axis for sewing so as to invert the staple line. As indicated by the learning curve(Fig. 2), it took us about 10 min to finish the purse-string at the early stage, because of the technical difficulty associated with the procedure. However, the time decreased to 5 min after we had 
finished 65 purse-string cases. This indicates that purse-string sutures could be easily applied during laparoscopic uncut Roux-en-Y reconstruction without increasing operation time drastically.

Nonetheless, this study was associated with certain limitations due to its retrospective design. Firstly, the study was not a prospective, randomized controlled trial, and there were some changes during the study period. Typically, the most important change was the surgeons' increasing experience in laparoscopic surgery, which would affect the outcomes.

\section{Conclusion}

Based primarily on the research, the incidence of duodenal stump leakage can be reduced by reinforcement with a hand-sewn purse-string suture as it is a serious complication after gastrectomy under laparoscope with Roux-en-Y or Billroth-II reconstruction.

\section{Abbreviations}

GC: Gastric cancer

NR: Non-reinforcement group

R: Reinforcement group

DSF: Duodenal stump fistula

LND: Lymph node dissection

AJCC: American Joint Committee on Cancer

SD: Standard deviation

BMl: Body mass index

\section{Declarations}

\section{Ethics approval and consent to participate}

Not applicable.

\section{Consent for publication}

Not applicable.

\section{Availability of data and material}


All data generated or analysed during this study are included in this published article.

\section{Competing interests}

Fengyuan Li, Jianghao Xu, Hao Xu, Weizhi Wang, Caidian Zhang, Li Yang, Linjun Wang, Yugang Ge, Yiwen Xia, Liang Shi, Lu Wang, Zekuan Xu have no conflicts of interest or financial ties to disclose.

\section{Funding}

This article is funded by Jiangsu Provincial key research and development program(BE2016786).

\section{Acknowledgements}

Not applicable.

\section{References}

1. Aurello P, Sirimarco D, Magistri P, Petrucciani N, Berardi G, Amato S, Gasparrini M, et al. Management of duodenal stump fistula after gastrectomy for gastric cancer: Systematic review. World J Gastroenterol. 2015;21:7571-6. doi:10.3748/wjg.v21.i24.7571.

2. Kim KH, Kim MC, Jung GJ. Risk factors for duodenal stump leakage after gastrectomy for gastric cancer and management technique of stump leakage. Hepatogastroenterology. 2014;61:1446-53. PMID: 25436323.

3. Hirao M, Takiguchi S, Imamura H, Yamamoto K, Kurokawa Y, Fujita J, et al. Comparison of Billroth I and Roux-en-Y reconstruction after distal gastrectomy for gastric cancer: one-year postoperative effects assessed by a multi-institutional RCT. Ann Surg Oncol. 2013;20:1591-7. doi:10.1245/s10434-012-2704-9.

4. Terashima M, Tanabe K, Yoshida M, Kawahira H, Inada T, Okabe H, et al. Postgastrectomy Syndrome Assessment Scale (PGSAS)-45 and changes in body weight are useful tools for evaluation of reconstruction methods following distal gastrectomy. Ann Surg Oncol. 2014;21:370-8. doi:10.1245/s10434-014-3583-z.

5. Kawahira H, Kodera Y, Hiki N, Takahashi M, Itoh S, Mitsumori N, et al. Optimal Roux-en-Y reconstruction after distal gastrectomy for early gastric cancer as assessed using the newly developed PGSAS-45 scale. Surg Today. 2015;45:1307-16. doi:10.1007/s00595-015-1111-9.

6. Kitano S, Iso Y, Moriyama M, Sugimachi K. Laparoscopy-assisted Billroth I gastrectomy. Surg Laparosc Endosc. 1994;4:146-8. doi:10.1097/sle.0000000000000011.

7. Cheng Q, Pang TC, Hollands MJ, Richardson AJ, Pleass H, Johnston ES, et al. Systematic review and meta-analysis of laparoscopic versus open distal gastrectomy. J Gastrointest Surg. 2014;18:1087- 
99. doi:10.1007/s11605-014-2519-z.

8. Uemura M, Yamashita M, Tomikawa M, Obata S, Souzaki R, leiri S, et al. Objective assessment of the suture ligature method for the laparoscopic intestinal anastomosis model using a new computerized system. Surg Endosc. 2015;29:444-52. doi:10.1007/s00464-014-3681-9.

9. Cozzaglio L, Coladonato M, Biffi R, Coniglio A, Corso V, Dionigi P, et al. Duodenal fistula after elective gastrectomy for malignant disease. An Italian retrospective multicentric study. J Gastrointest Surg. 2010;14:805-11. doi:10.1007/s11605-010-1166-2.

10. Babu BI, Finch JG. Current status in the multidisciplinary management of duodenal fistula. Surgeon. 2013;11:158-64. doi:10.1016/j.surge.2012.12.006.

11. Cozzaglio L, Cimino M, Mauri G, Ardito A, Pedicini V, Poretti D, et al. Percutaneous transhepatic biliary drainage and occlusion balloon in the management of duodenal stump fistula. J Gastrointest Surg. 2011;15:1977-81. doi:10.1007/s11605-011-1668-6.

12. Khairy GE, al-Saigh A, Trincano NS, al-Smayer S, al-Damegh S. Percutaneous obliteration of duodenal fistula. J R Coll Surg Edinb. 2000;45:342-4. PMID: 11077786.

13. Bianchi A, Solduga C, Ubach M. Percutaneous obliteration of a chronic duodenal fistula. Br J Surg. 1988;75:572. doi:10.1002/bjs.1800750624.

14. Bini R, Coppola F, Recchia S, Fusca M, Gaia S, Leli R. Endoscopic treatment of postgastrectomy duodenal fistula with an over-the-scope clip. Surg Innov. 2011;18:102-4. doi:10.1177/1553350610392244.

15. Curcio G, Badas R, Miraglia R, Barresi L, Tarantino I, Traina M. Duodenal stump fistula following Roux-en-Y gastrectomy, treated with single-balloon enteroscopy using the tulip bundle technique and fibrin glue injection. Endoscopy. 2012;44:E364-5. doi:10.1055/s-0032-1310073.

16. Cozzaglio L, Farinella E, Coladonato M, Sciannameo F, Gennari L, Doci R. Current role of surgery in the treatment of digestive fistulas. Ann Ital Chir. 2010;81:285-94. PMID: 21319701.

17. Bo T, Zhihong P, Peiwu Y, Feng Q, Ziqiang W, Yan S, et al. General complications following laparoscopic-assisted gastrectomy and analysis of techniques to manage them. Surg Endosc. 2009;23:1860-5. doi:10.1007/s00464-008-0312-3.

18. Bracale U, Marzano E, Nastro P, Barone M, Cuccurullo D, Cutini G, et al. Side-to-side esophagojejunostomy during totally laparoscopic total gastrectomy for malignant disease: a multicenter study. Surg Endosc. 2010;24:2475-9. doi:10.1007/s00464-010-0988-z.

19. Kumagai K, Hiki N, Nunobe S, Jiang X, Kubota T, Aikou S, et al. Different features of complications with Billroth-I and Roux-en-Y reconstruction after laparoscopy-assisted distal gastrectomy. J Gastrointest Surg. 2011;15:2145-52. doi:10.1007/s11605-011-1683-7.

20. Kang KC, Cho GS, Han SU, Kim W, Kim HH, Kim MC, et al. Comparison of Billroth I and Billroth II reconstructions after laparoscopy-assisted distal gastrectomy: a retrospective analysis of large-scale multicenter results from Korea. Surg Endosc. 2011;25:1953-61. doi:10.1007/s00464-010-1493-0.

21. Lee IS, Kim TH, Kim KC, Yook JH, Kim BS. Modified techniques and earlyoutcomes of totally laparoscopic total gastrectomy with sideto-side esophagojejunostomy. J Laparoendosc Adv Surg 
Tech A. 2012;22:876-80. doi:10.1089/lap.2012.0177.

22. Zhang C, Xiao W, Chen K, Zhang Z, Du G, Jiang E, et al. A new intracorporeal Billroth II stapled anastomosis technique in totally laparoscopic distal gastrectomy. Surg Endosc. 2015;29:1636-42. doi:10.1007/s00464-014-3825-y.

23. Okabe H, Obama K, Tsunoda S, Tanaka E, Sakai Y. Advantage of completely laparoscopic gastrectomy with linear stapled reconstruction: a long-term follow-up study. Ann Surg. 2014;259:109-16. doi:10.1097/SLA.0b013e31828dfa5d.

24. Kim DJ, Lee JH, Kim W. Comparison of the major postoperative complications between laparoscopic distal and total gastrectomies for gastric cancer using Clavien-Dindo classification. Surg Endosc. 2015;29:3196-204. doi:10.1007/s00464-014-4053-1.

25. Jiang X, Hiki N, Nunobe S, Nohara K, Kumagai K, Sano T, et al. Laparoscopy-assisted subtotal gastrectomy with very small remnant stomach: a novel surgical procedure for selected early gastric cancer in the upper stomach. Gastric Cancer. 2011;14:194-9. doi:10.1007/s10120-011-0023-7.

\section{Figures}
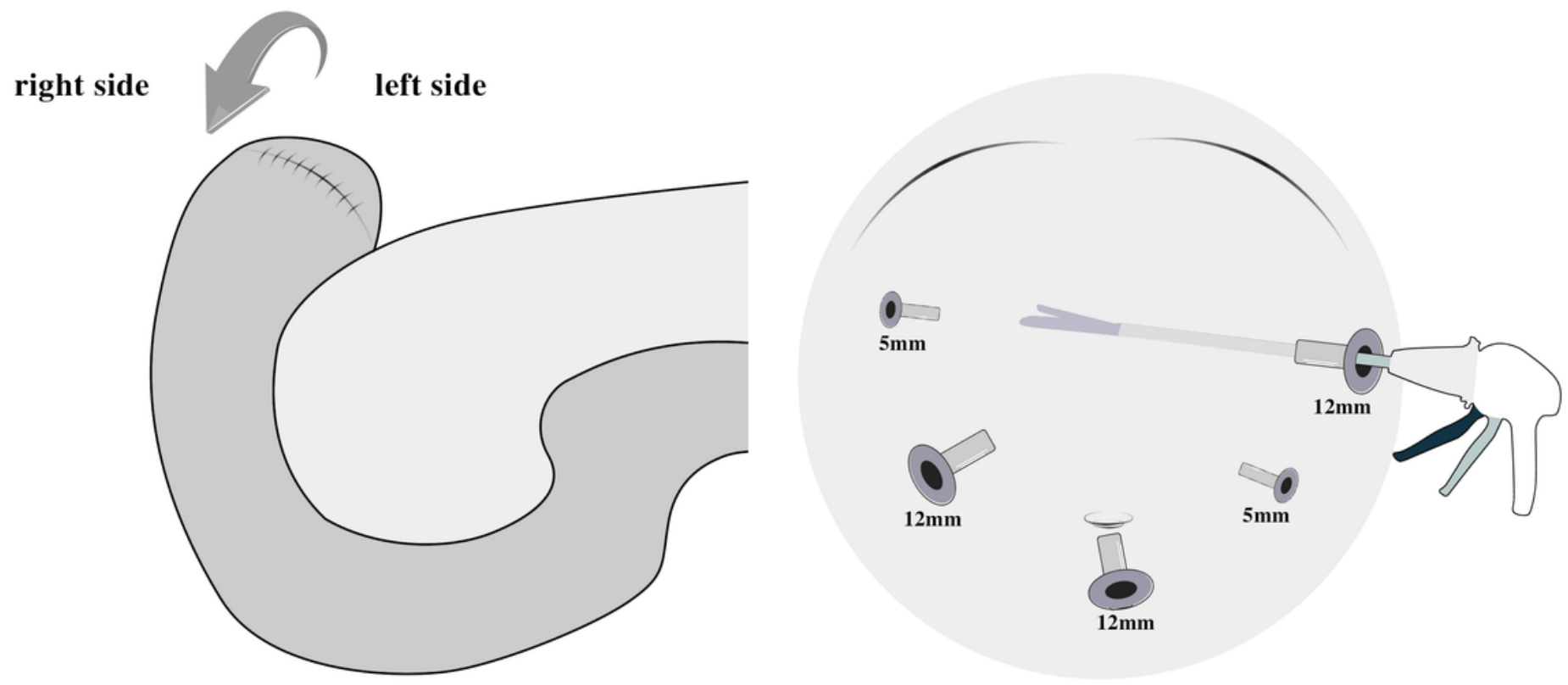

\section{Figure 1}

5 trocars were used for the surgical procedure for all patients. The surgeon stood at the patient's left side, while the camera assistant stood between the patient's legs., The duodenum was cut using a $60 \mathrm{~mm}$ endoscope-assisted linear stapler through the trocar in the left upper side with a single white cartridge. In addition, in group $\mathrm{R}$, the duodenal staple line would be reinforced by a hand-sewn purse-string suture (continuous muscular absorbable 3-0 sutures). 


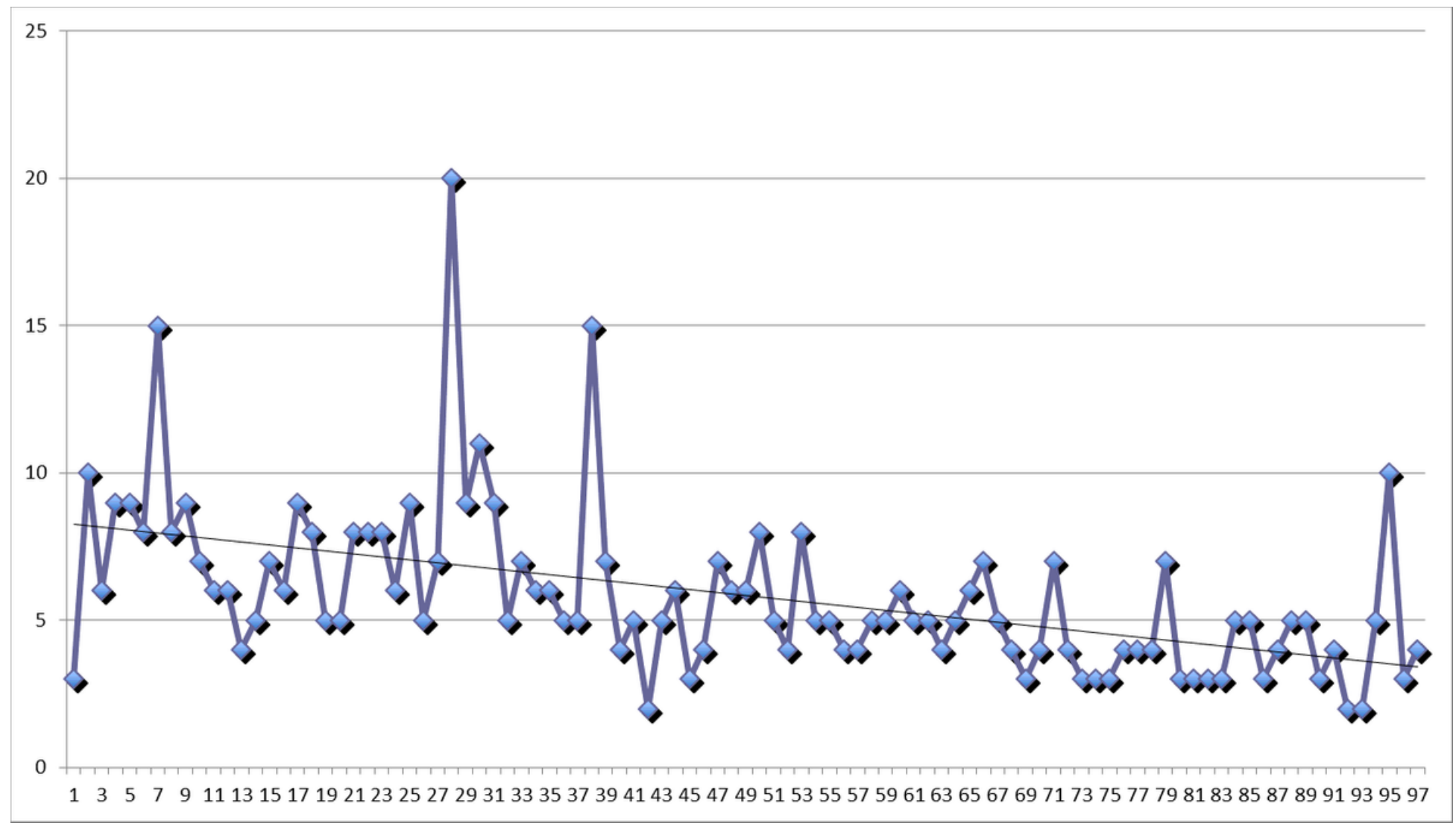

Figure 2

At the early stage, It took about 10 minutes to finish the purse-string. However, the time decreased to 5 min after 65 purse-string cases finished. 